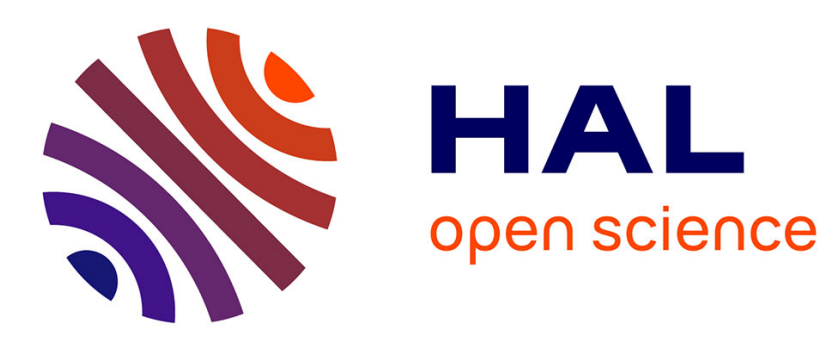

\title{
On the mean field theory of mixed spin glass-ferromagnetic phases
}

\author{
G. Toulouse
}

\section{To cite this version:}

G. Toulouse. On the mean field theory of mixed spin glass-ferromagnetic phases. Journal de Physique Lettres, 1980, 41 (18), pp.447-449. 10.1051/jphyslet:019800041018044700 . jpa-00231818

\section{HAL Id: jpa-00231818 https://hal.science/jpa-00231818}

Submitted on 1 Jan 1980

HAL is a multi-disciplinary open access archive for the deposit and dissemination of scientific research documents, whether they are published or not. The documents may come from teaching and research institutions in France or abroad, or from public or private research centers.
L'archive ouverte pluridisciplinaire HAL, est destinée au dépôt et à la diffusion de documents scientifiques de niveau recherche, publiés ou non, émanant des établissements d'enseignement et de recherche français ou étrangers, des laboratoires publics ou privés. 


\title{
On the mean field theory of mixed spin glass-ferromagnetic phases
}

\author{
G. Toulouse \\ Laboratoire de Physique de l'Ecole Normale Supérieure, 24, rue Lhomond, 75231 Paris Cedex 05, France
}

(Reçu le 13 juin 1980, accepté le 25 juillet 1980)

\begin{abstract}
Résumé. - Les propriétés thermodynamiques du modèle à portée infinie, en présence d'une interaction ferromagnétique moyenne $\left(J_{0} \neq 0\right)$, se déduisent aisément du cas $J_{0}=0$. Les conséquences de certaines propriétés subtiles, prédites pour la phase verre de spin, apparaissent alors en pleine lumière.
\end{abstract}

Abstract. - The thermodynamic properties of the infinite-ranged model, in the presence of a mean ferromagnetic interaction $\left(J_{0} \neq 0\right)$, are easily derived from the case $J_{0}=0$. The consequences of some subtle properties, predicted for the spin glass phase, are then brought into full light.

1. Introduction. - In order to obtain a mean field theory of spin glasses, one studies the infinite-ranged Sherrington-Kirkpatrick (SK) model [1] :

$$
\mathscr{H}=-\sum_{(i j)} J_{i j} S_{i} \cdot S_{j}-H \sum_{i} S_{i}
$$

where the $J_{i j}$ are independent random variables. Among all the cumulants of the random law $p\left(J_{i j}\right)$, only the first $J_{0} / N$ and the second $J^{2} / N$ are relevant, due to the normalization ( $N$ is the number of spins) which must be enforced to obtain an appropriate thermodynamic limit. Therefore, the problem has four natural parameters : $T, H, J_{0}, J$; setting $J=1$ as energy scale, one is left with three parameters : $T, H, J_{0}$.

Though a simple exercise really, it is nevertheless instructive to consider how the properties of the SK model with an average ferromagnetic interaction $\left(J_{0} \neq 0\right)$ can be read off from the properties of the model without $\left(J_{0}=0\right)$. The simple trick is based on the fact that the energy contribution (per spin) coming from a non-zero $J_{0}$ is :

$$
-J_{0} \frac{M^{2}}{2}
$$

where $M$ is the magnetization (per spin). This is a direct consequence of the infinite range of the interactions, and illustrates the mean field character of the model. Thus the energy (per spin) may be written :

$$
U\left(H, J_{0}\right)=U\left(H=0, J_{0}=0\right)-H M-J_{0} \frac{M^{2}}{2} .
$$

This shows clearly that the extensive variables, conjugate to the intensive parameters $H$ and $J_{0}$, are not independent. Then one reckons that a thermodynamic potential, function of $T$ and $M$, will be the right potential to work with.

Indeed, if this thermodynamic potential is called $A(T, M)$, in the absence of an average ferromagnetic interaction $\left(J_{0}=0\right)$, then it will simply be :

$$
A(T, M)-J_{0} \frac{M^{2}}{2}
$$

in the presence of a finite $J_{0}$. From this expression and the differential identity :

$$
\mathrm{d} A=H \mathrm{~d} M-S \mathrm{~d} T
$$

all thermodynamic quantities, for $J_{0}$ finite, can be obtained from a knowledge of $A(T, M)$.

Since the true behaviour of the SK model, for $J_{0}=0$, is not yet completely unravelled, it may appear that the procedure indicated above is for the moment just another way of tourner autour du pot. However, there exist some rather educated guesses on the $J_{0}=0$ case, and the introduction of a finite $J_{0}$ helps to unfold neatly some of their detailed analytical consequences.

2. The phase diagram. - A schematic plot is given in figure 1, where the transition lines occurring in the planes $(H, T)$ and $\left(J_{0}, T\right)$ are depicted and numbered, for clarity purposes. There is actually a transition surface in the quadrant $\left(T, H, J_{0}\right)$ bordered by lines (1), 


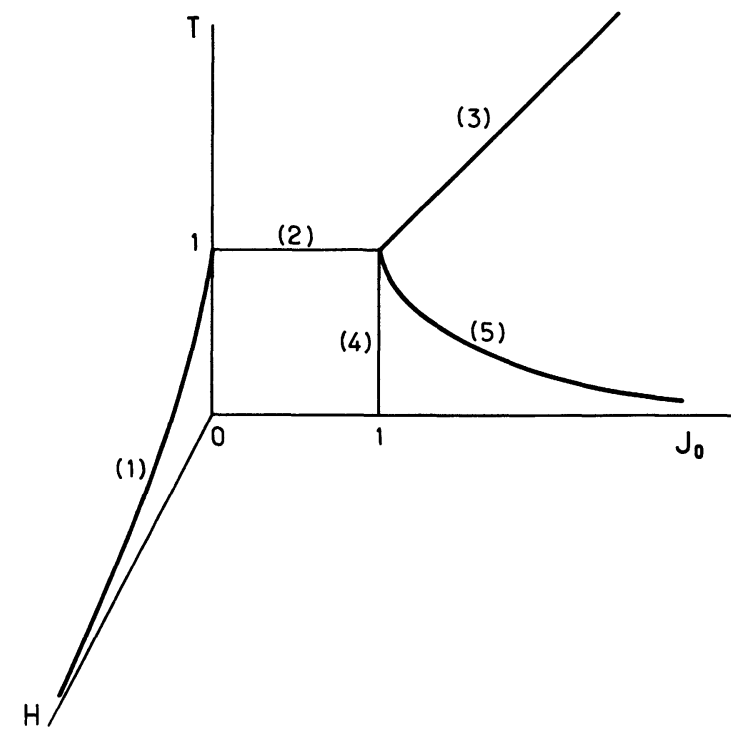

Fig. 1. - Phase diagram of the Ising SK model, as a function of temperature $T$, average ferromagnetic interaction $J_{0}$ and magnetic field $H$.

(2) and (5), corresponding to the breaking of replica symmetry [2]. Lines (3) and (4) are isolated (the transition disappears in finite field $H$ ) and correspond to the onset of ferromagnetic order.

We are going to show how the shapes of these transition surfaces and lines, and the nature of the transitions across them, can be derived from a knowledge of the case $J_{0}=0$. For this case, we assume the validity of the Parisi-Toulouse hypothesis [3] (heretofore called the PaT hypothesis), which has the virtue of producing clear-cut predictions and of reproducing many of the findings of the Parisi replicasymmetry-breaking scheme [4].

For $T>1$, the thermodynamic potential $A(T, M)$ is a regular function of $M$ :

$$
A(T, M)=A_{0}(T)+T \frac{M^{2}}{2}+0\left(M^{4}\right),
$$

yielding a Curie law for the susceptibility.

For $T<1$, the potential $A(T, M)$ has a singularity at a value of $M$ :

$M=M_{\mathrm{c}}(T)$

for $M<M_{\mathrm{c}}(T)$, the PaT hypothesis tells that :

with

$$
A(T, M)=A_{1}(T)+A_{2}(M),
$$

$$
A_{2}(M)=\frac{M^{2}}{2}+\frac{3}{10}\left(\frac{3}{4}\right)^{2 / 3} M^{10 / 3}+0\left(M^{4}\right) ;
$$

implying the existence of another singularity at the origin $(M=0)$.

The value $M_{\mathrm{c}}(T)$ and the position $H_{\mathrm{c}}(T)$ of the instability line (number (1) in Fig. 1) can be calculated with arbitrary precision, since no replica symmetry breaking is necessary for that. To lowest order :

$$
M_{\mathrm{c}}(T) \simeq H_{\mathrm{c}}(T) \simeq\left(\frac{4}{3}\right)^{1 / 2}(1-T)^{3 / 2}, \text { for } T \lesssim 1,
$$

whereas for $T$ small,

$$
\left\{\begin{array}{l}
M_{\mathrm{c}}(T) \sim 1-\frac{3 T}{2}\left[2 \log \left(\frac{3}{2 T} \sqrt{\frac{2}{\pi}}\right)\right]^{1 / 2}, \\
T \sim \frac{2}{3} \sqrt{\frac{2}{\pi}} \cdot \exp \left(-\frac{H_{\mathrm{c}}^{2}(T)}{2}\right) .
\end{array}\right.
$$

Now, the position of the transition surface, corresponding to instability of the replica symmetry, is given by :

$$
H+J_{0} M_{\mathrm{c}}(T)=H_{\mathrm{c}}(T),
$$

an equation involving the three parameters $T, H, J_{0}$. For $H \rightarrow 0$, one obtains the equation of line (2) :

$$
T=1, \quad 0 \leqslant J_{0} \leqslant 1,
$$

and the equation of line (5):

$$
\begin{cases}J_{0}-1 \sim(1-T)^{2}, & \text { for } T \lesssim 1, \\ T \sim \frac{2}{3} \sqrt{\frac{2}{\pi}} \exp \left(-\frac{J_{0}^{2}}{2}\right), & \text { for } T \text { small } .\end{cases}
$$

Finally, the verticality of line (4) is a straightforward consequence of the expression for $A(T, M)$ given above. Since $A_{2}(M)$ is temperature independent in the spin glass phase, the coefficient of $M^{2}$, in

$$
A(T, M)-J_{0} \frac{M^{2}}{2},
$$

vanishes for $J_{0}=1$, at any temperature smaller than $T=1$. The crucial point in this argument is the temperature-independence of the (zero-field) susceptibility $\chi$ in the spin glass phase, for $J_{0}=0$. In a recent meeting (Antwerpen, April 1980), M. Kosterlitz [5] mentioned to us that he had made low temperature expansions which agreed with a vertical line (4) and that moreover $D$. Thouless had a thermodynamic argument for it. So this result is not presented here with a claim of being original. However, the use of the PaT hypothesis allows to go further and to make predictions for the nature of the transition singularities.

3. Nature of the transitions. - One may distinguish two sets of transition lines. Across transition lines (2) and (3), there is not much problem. The transitions across lines (4) and (5) offer more interest.

Across line (2), the transition is quite similar to what occurs for $J_{0}=0$. The only notable difference is that there is a renormalization of the field-dependent quantities. For instance, the zero-field susceptibility is :

$$
\chi=\frac{1}{1-J_{0}} .
$$


Line (3) is a standard second order ferromagnetic transition line.

Around transition line (4), the thermodynamic potential is obtained as :

$$
\begin{aligned}
A(T, M)-J_{0} \frac{M^{2}}{2}= & A_{1}(T)+\frac{M^{2}}{2}\left(1-J_{0}\right)+ \\
& +\frac{3}{10}\left(\frac{3}{4}\right)^{2 / 3} M^{10 / 3}+0\left(M^{4}\right) .
\end{aligned}
$$

Therefore, the spontaneous magnetization is predicted to vary as :

$$
M \sim\left(J_{0}-1\right)^{3 / 4}, \text { for } J_{0} \gtrsim 1,
$$

a power law behaviour with an unusual exponent. For $J_{0}=1$, the stability line is given by :

$$
H_{\mathrm{c}}\left(J_{0}=1, T\right) \sim\left(\frac{4}{3}\right)^{1 / 2}(1-T)^{7 / 2}, \text { for } T \lesssim 1,
$$

and the magnetization, in low field, is predicted to vary as :

$$
M \sim\left(\frac{4}{3}\right)^{2 / 7} H^{3 / 7}
$$

All these awkward exponents have been derived from the exponent $10 / 3$ in the PaT expression for $A_{2}(M)$. The existence of a finite $J_{0}$ has allowed the cancellation of the dominant $\boldsymbol{M}^{2}$ term and to bring to the fore the, usually subdominant, anomalous term. Also note that this term suggests, according to loose but swift arguments [6], an upper critical dimensionality $d_{\mathrm{c}}=5$ for the pure spin glass-mixed phase transition.

Transition line (5), and actually the whole instability surface, exhibits the same singularities as transition line (1). Within the PaT hypothesis, transition line (1) is predicted to be second order, in the Ehrenfest sense, with jumps for the specific heat and the susceptibility. This emphasizes, once more, the necessary relation, in this model, between the existence of a mixed phase, for $J_{0}>1$, and the existence of a spin glass phase,

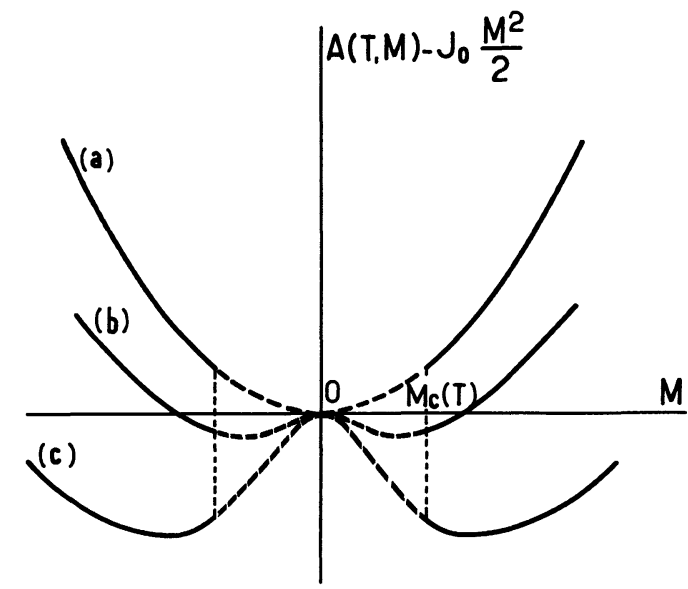

Fig. 2. - Sketch of the shape of the thermodynamic potential $A(T, M)-J_{0} \frac{M^{2}}{2}$, at a given temperature $T<1$, for three different values of $J_{0}$ : (a) $J_{0}=0$; (b) $J_{0} \gtrsim 1$; (c) $J_{0}$ large

for $J_{0}=0$. And the nature of the singularities on the instability line can be equivalently studied in the case $\left(J_{0}=0, H\right.$ finite) or in the case $\left(H=0, J_{0}\right.$ finite).

In order to illustrate this point, the thermodynamic potential $A(T, M)-J_{0} \frac{M^{2}}{2}$ has been sketched, in figure 2, for three different values of $J_{0}$, at a given temperature $T<1$. In zero field $H$, the minima of this potential give the equilibrium state which is, respectively, a pure spin glass, a mixed phase or a pure ferromagnetic state.

As a final comment, comparison of figure 1 of this paper and that of reference [7] is suggestive of what may happen for $\mathrm{m}$-component vector spins.

4. Conclusion. - Although the infinite-ranged SK model is obviously far from the physical reality of the materials accessible to the experimentalist, the results presented above do provide some further theoretical motivations for studying the mixed (ferromagneticspin glass) phase and its transitions toward pure ferromagnetic or pure spin glass phases.

\section{References}

[1] Sherrington, D., KirkPatrick, S., Phys. Rev. Lett. 32 (1975) 1792 ;

KirKPATriCK, S., SherRington, D., Phys. Rev. B 17 (1978) 4384

[2] De Almeida, J. R. L., Thouless, D. J., J. Phys. A 11 (1978) 983.

[3] ParisI, G., Toulouse, G., Submitted to J. Physique Lett.
[4] Parisi, G., J. Phys. A 13 (1980) 1101.

[5] Kosterlitz, J. M., Private communication.

[6] Toulouse, G., Nuovo Cimento 23 B (1974) 234.

[7] Kosterlitz, J. M., Thouless, D. J., Jones, R. C., Phys. Rev. Lett. 36 (1976) 1217. 